\title{
¿La vida en pausa? Impacto de la COVID-19 en la vida de jóvenes estudiantes de educación superior que retornan al ámbito rural
}

\author{
Alexia Potesta Cortez \\ Estudiante de Antropología de la Pontificia Universidad Católica del Perú (PUCP). \\ e-mail: apotesta@pucp.edu.pe
}

\section{Luciana Ramírez Sánchez}

Estudiante de Antropología de la Pontificia Universidad Católica del Perú (PUCP). e-mail: ramirezs.1c@pucp.pe

\section{Mauricio Alarcón Piña}

Estudiante de Antropología de la Pontificia Universidad Católica del Perú (PUCP). e-mail: mauricio.alarconp@pucp.edu.pe

\section{María Luisa Pastor Castillo}

Estudiante de Antropología de la Pontificia Universidad Católica del Perú (PUCP). e-mail:mpastorc@pucp.edu.pe

\section{Resumen}

El presente artículo explora el proceso de migración de retorno de jóvenes peruanos hacia espacios rurales y ciudades intermedias en el contexto de la pandemia. En específico, se enfoca en la nueva convivencia familiar, las dificultades con la educación virtual, y los cambios o permanencias en sus proyectos de vida durante la pandemia de la COVID-19. En un primer momento, se explican los cambios en las dinámicas familiares, la medida en que las nuevas responsabilidades impactan en su vida cotidiana y las valoraciones positivas en cuanto a la nueva convivencia. Posteriormente, se propone comprender las experiencias y cambios ocurridos debido a la virtualización de la educación superior, con énfasis en las limitaciones en infraestructura y tecnología, presentes en las zonas rurales. Finalmente, se busca analizar de qué manera la pandemia por la COVID-19 fortaleció y transformó ciertos aspectos de sus proyectos de vida. Para la presente investigación, se empleó una metodología cualitativa exploratoria, basada en entrevistas realizadas de manera virtual a 16 jóvenes retornantes a ciudades intermedias y a espacios rurales, 10 mujeres y 6 hombres, de un rango de edad de 18 a 26, en el marco de un trabajo de campo virtual realizado entre octubre y noviembre del 2020.

\section{Palabras clave}

Pandemia, migración de retorno, proyectos de vida, juventud rural.

Nota editorial: el presente artículo fue recibido el 17-01-21 y aprobado el 08-02-21. 


\title{
Life on hold? Impact of COVID-19 on the lives of young higher education students returning to rural areas
}

\author{
Alexia Potesta Cortez \\ Anthropology student at the Pontifical Catholic University of Peru (PUCP). \\ e-mail: apotesta@pucp.edu.pe \\ Luciana Ramírez Sánchez \\ Anthropology student at the Pontifical Catholic University of Peru (PUCP). \\ e-mail: ramirezs.lc@pucp.pe \\ Mauricio Alarcón Piña \\ Anthropology student at the Pontifical Catholic University of Peru (PUCP). \\ e-mail: mauricio.alarconp@pucp.edu.pe \\ María Luisa Pastor Castillo \\ Anthropology student at the Pontifical Catholic University of Peru (PUCP). \\ e-mail: mpastorc@pucp.edu.pe
}

\begin{abstract}
This article explores the return migration of young Peruvians to rural areas and intermediate cities. Specifically, we focus on the new family coexistence, the difficulties with virtual education, and the changes or permanence in their life projects during the COVID-19 pandemic. At first, we investigate the changes in family dynamics, the extent to which the new responsibilities impact on their daily life and the positive evaluations regarding the new coexistence. Furthermore, we address the experiences and changes arising from the virtualization of higher education, with an emphasis on the limitations in infrastructure and technology present in rural areas. Finally, this essay will seek to analyze how the COVID-19 pandemic strengthened and transformed certain aspects of their life projects. For the present research, an exploratory qualitative methodology was used, based on virtual interviews with 16 young people returning to intermediate cities and rural areas, 10 women and 6 men, with an age range of 18 to 26 , within the framework of a virtual fieldwork carried out between October and November of 2020.
\end{abstract}

\section{Keywords}

Pandemic, return migration, rife projects, rural youth. 


\section{Introducción}

La pandemia global de la COVID-19 ha tenido consecuencias en la población peruana, no solo a nivel de salud, sino también a nivel económico, aunque el impacto ha sido diferenciado en función del género, la clase, la residencia y la edad. Uno de los fenómenos más llamativos relacionados a ello fueron las movilizaciones de retorno ocurridas durante el periodo inicial de la pandemia en el país, que implicaron el desplazamiento de buena parte de la población migrante urbana al área rural. Entre el grupo de los llamados "retornantes", se destaca la presencia de jóvenes, quienes volvieron a sus pueblos debido a distintas circunstancias. Para entenderlo, es necesario tener en cuenta que la migración entre el campo y la ciudad constituye una estrategia vital para personas que buscan acceder a educación superior en universidades o institutos localizados en ciudades, con el fin de ascender socialmente y lograr autonomía (Trivelli y Urrutia, 2018). Para lograrlo, se suele recurrir a estrategias de apoyo por parte de distintas redes familiares que permiten dejar el espacio doméstico y asentarse en la ciudad (Trivelli y Urrutia, 2018). Ahora bien, en el contexto de la pandemia, muchos jóvenes han tenido que retornar a sus lugares de origen por la crisis económica y sanitaria. Este retorno ha implicado una nueva convivencia dentro del espacio doméstico con nuevos desafíos para que los jóvenes puedan continuar sus estudios de manera virtual. Incluso, la misma virtualidad representa un reto para los jóvenes, debido a las brechas relativas en el acceso a servicios básicos, como electricidad o internet, lo cual tiene el potencial de agudizar el estado de desigualdad en la educación previo a la pandemia (Proyecto CRECER, 2020). Asimismo, esta migración de retorno puede tener implicancias para los proyectos de vida que se habían trazado los jóvenes antes de la pandemia, si se tiene en cuenta que en situaciones de crisis social, la incertidumbre y la frustración pueden producir conmociones y revalorizaciones importantes de los proyectos de vida individuales y colectivos (D'Angelo, 2004).

De esta manera, se hace necesario comprender de qué modo los jóvenes han sido afectados por la pandemia; sobre todo, en lo concerniente a las nuevas dinámicas familiares y los cambios surgidos en la educación superior por la virtualización -ya que estos dos aspectos, a su vez, pueden tener un impacto considerable en sus propios planes de vida. Al entender qué ocurre con este aspecto que debe ser abordado, será posible tomar medidas políticas estatales y políticas públicas más efectivas con respecto a los jóvenes en el marco de la pandemia y a futuro. Además, al ser un tema reciente, no existen aún estudios que consideren una perspectiva antropológica de esta situación. Por ello, en el presente trabajo se busca explorar los modos en que la pandemia por la COVID-19 impacta en la vida familiar, educativa y personal de los jóvenes retornantes. La presente investigación es de tipo cualitativa exploratoria y se basa en entrevistas realizadas de manera virtual en el marco de un trabajo de campo virtual realizado entre los meses de octubre y noviembre del 2020. Dichas entrevistas se dieron por medio de mensajes y videollamadas a dieciséis estudiantes retornantes, diez mujeres y seis hombres, de un rango de edad entre 18 a 26 años. 
Estos se movilizaron desde ciudades como Trujillo, Huaraz, Lima, Huánuco, Cusco y Junín hacía ciudades intermedias ${ }^{1}$ y espacios rurales ${ }^{2}$. Los jóvenes accedieron a ser entrevistados gracias a que se les presentó un documento firmado por la PUCP. Asimismo, algunos de ellos pidieron que su privacidad sea resguardada, por lo cual hemos usado seudónimos en el caso de algunos.

\section{Aproximación teórica y conceptos clave}

Uno de los conceptos importantes para enmarcar esta investigación es el de transición hacia la adultez. Por ello, se entiende el paso de la juventud a la adultez teaniendo en cuenta que este tránsito es diverso. Lo que idealmente define ese paso es la adquisición de autonomía respecto a los adultos de los cuales se depende. Sin embargo, los cambios en el capitalismo de las últimas décadas han generado una mayor falta de estabilidad económica y de precarización del trabajo (Harvey, 2007). Por tal razón, si bien la búsqueda de autonomía es un proyecto, este debe ser constantemente reformulado de acuerdo a los cambios en el mercado, lo cual produce una diversidad de estrategias (Punch, 2015). Ello es particularmente preciso en países en vías de desarrollo, pues, a diferencia de los países desarrollados donde tiende a haber una transición lineal (de la escuela a la universidad y de la universidad al trabajo) y tardía, en los países subdesarrollados esta transición hacia el campo laboral puede ser más temprana y plurilineal, más aún con las brechas existentes según el género, la clase, la etnicidad y la residencia ${ }^{3}$. En ese contexto, la motivación para la migración de estos jóvenes suele implicar también la búsqueda de integración en la "sociedad oficial" y la adquisición de status (Azaola, 2012). Asimismo, ante la inestabilidad en la que se encuentran, sus estrategias de transición a la adultez se mueven entre la negociación y la imposición, modificando sus planes y estrategias de acuerdo a las oportunidades que se les presentan ${ }^{5}$. No obstante, entre todas estas estrategias, las prioridades son la educación y el trabajo, y la migración con esos fines para los jóvenes de sectores rurales. Por ello, cobran principal relevancia en la migración las relaciones de parentesco y las expectativas de estos sobre los jóvenes en el modo de estructurar sus procesos de transición (Punch, 2015).

Otro concepto relevante y relacionado con el primero es el de estrategias de vida. Con ello, se entiende a "las capacidades, valores y actividades de las familias campesinas para proveerse sus medios de vida" (Conway y Chambers, 1992, p. 5). Comprende los

\footnotetext{
Huaraz (Áncash), Barranca (Lima), San Vicente de Cañete (Lima).

Chavín de Pariarca (Huánuco), Caraz (Áncash), Cascapara (Áncash), Chacas (Áncash), Chiquian (Áncash), Vijus (La Libertad), Coñamuro (Cusco), Paucartambo (Cusco), Kiteni (Cusco), Chirimoto (Amazonas), Acolla (Junin).

3 En el área rural, un 16,3\% de mujeres y un 16,0\% de hombres se matriculan en educación superior, en contraste con un el 35,9 \% de las mujeres y el 33,6\% de hombres del área urbana, que constituye casi el doble (INEI, 2019)

4 Es decir, la situación de marginación en la que vive gran parte de la población rural busca ser superada a través de la migración a la ciudad con fines de trabajo o estudio.

5 Ello lleva a que los planes a largo plazo sean más generales, lo que se evidencia en expresiones tales como "salir adelante".
} 
activos o capitales (naturales, físicos, humanos, financieros o sociales) que disponen las familias, las actividades que desarrollan y el acceso a recursos y medios de producción (mediado por instituciones y relaciones sociales). La migración es así parte de esta estrategia mayor, como se evidencia en las estrategias de "doble residencia", en las cuales miembros de una misma familia nuclear rural se encuentran viviendo fuera del espacio local (usualmente en una ciudad cercana) mientras que los otros miembros residen en el mismo espacio local, intercalando sus lugares de residencia con temporalidad y fluidez variable. Esta doble residencia busca aprovechar distintas actividades productivas, lo cual puede ser tanto una estrategia de acumulación de capital como una alternativa temporal de generación de ingresos. El desplazamiento entre ambas residencias depende de las oportunidades y condiciones externas (Koc, 2001). En nuestra investigación, la doble residencia era una estrategia que tenía como objetivo principal el que los jóvenes puedan estudiar en la ciudad mientras sus padres los abastecían económicamente desde su lugar de origen. Además, el capital social ${ }^{6}$ es muy importante en estos contextos, pues las redes -de parientes o amigos- permiten el acceso a ciertos recursos en las ciudades. Así, estas redes permiten el acceso a puestos de trabajo, a información relevante, a otros contactos, a vivienda, etc. Sin embargo, estas redes no son solo "recursos" a ser utilizados estratégicamente por los actores, sino que estas generan al mismo tiempo obligaciones e influencian en sus propias prácticas y aspiraciones. Finalmente, estas estrategias se enmarcan en desigualdades estructurales en función del género, el grado de ruralidad de la residencia y la clase social que se imponen y, al mismo tiempo, negocian con las aspiraciones de los jóvenes entrevistados.

Por último, es necesario entender el concepto de emprendedurismo, utilizado como sinónimo de una filosofía de vida encaminada al éxito económico individual. Según Lamas (2017), su influencia en el discurso público del Perú en los últimos años es notoria. Cada vez con más frecuencia es posible detectar mensajes que apelan a esta filosofía individualista, la cual legitima distinciones y restricciones sobre quienes no ejercitan su "libertad" dentro de los marcos establecidos por el sistema. Este pensamiento celebra la capacidad del individuo para abrirse camino en la vida, por medio del éxito económico personal. En ese sentido, el emprendedor se convierte en el modelo ideal de ciudadano, pues es aquel que usa responsable y racionalmente su libertad para aumentar sus recursos: es un "empresario de sí mismo" (Lamas, 2017, p. 56). En el caso de la mayoría de nuestros entrevistados, se evidencia la presencia de este discurso emprendedor que acompaña sus proyectos de vida a largo a plazo, debido a que estos buscan independizarse, tener un ingreso propio y mejorar sus condiciones de vida gracias a la educación que reciben o a un negocio propio que se pueda formar en un futuro. En muchos casos, la educación universitaria es un medio para lograr este fin.

\footnotetext{
6 Para Bourdieu (2000) el capital se construye en base a diferentes acciones, algunas aprendidas casi sin ser tan conscientes de ellas y otras con una intencionalidad más clara. El manejo de cierto capital en ciertos contextos produce beneficios lo que hace que este capital se reproduzca. En ese sentido, el capital social se refiere a las relaciones que se establecen con otras personas, redes sociales, alianzas y su posterior gestión, que permite el logro de objetivos personales.
} 


\section{Dinámicas familiares y nueva convivencia en la pandemia}

Entre la mayoría de los entrevistados, existe una historia migratoria similar: muchos de ellos son los primeros en su línea familiar en migrar a una ciudad para acceder a la educación superior. Debido a las brechas existentes en educación superior entre el campo y la ciudad, la migración a esta última constituye una vía de ascenso social, tanto para los entrevistados como para sus familias. Por ello, el apoyo de redes familiares es fundamental para el desarrollo de la educación superior de los jóvenes migrantes como una estrategia colectiva de movilidad social. Este apoyo consiste, sobre todo, en lo relativo a vivienda y alimentación durante su estadía en la ciudad, así como el apoyo monetario de los padres para la manutención mensual. En algunos casos, además, las redes de ayuda por parte de parientes involucran ayudas en la obtención de trabajo en negocios familiares o el apoyo con la preparación para la postulación a la universidad.

El apoyo brindado por parte de las redes familiares se pudo evidenciar, también, durante la crisis sanitaria por la COVID-19. La pandemia, y la consecuente crisis, obligó a nuestros entrevistados a movilizarse hacia sus lugares de origen como una medida temporal de supervivencia ante esa situación. Ahora bien, es importante recalcar que, en algunos casos, dado que la cuarentena comenzó en el periodo de vacaciones, los estudiantes ya se encontraban en el campo, entonces tomaron la decisión de quedarse ante el temor de que la situación sanitaria empeorara. Los entrevistados no tuvieron muchas dificultades en su viaje de retorno cuando se decretó la inmovilización social, debido a que, por un lado, tuvieron el apoyo económico de sus padres y, por otro lado, tuvieron el apoyo de otras redes que les brindaron la información necesaria para retornar o facilidades con el transporte. Asimismo, en el lugar de origen, encontraron un destino seguro frente a la incertidumbre por la crisis, pues contaban con el respaldo de su familia nuclear tanto en lo concerniente a vivienda como alimentación y otros cuidados necesarios para continuar con sus estudios.

Las familias de los entrevistados se dedican a la pequeña agricultura, ganadería y, en algunos casos, tienen negocios familiares ligados al sector de servicios, como tiendas, restaurantes $\mathrm{u}$ hoteles, y en un caso trabajan en la mina. Con el retorno de los estudiantes al campo, muchos de ellos se han visto ante la obligación de asumir responsabilidades y apoyar a sus padres en el trabajo agrícola, ganadero o doméstico; sin embargo, para otros jóvenes, este apoyo es secundario y hasta voluntario, debido a que los estudios son su actividad principal. La diferencia en el grado de obligatoriedad en la asunción de responsabilidades se debe a distintos factores, como el género -las mujeres suelen asumir mayores responsabilidades domésticasy la economía familiar - o el tipo de actividad de sustento de la familia- el trabajo agrícola demanda mayor cantidad de mano de obra que otros negocios familiares u otros oficios de los padres. Además, el grado de necesidad económica varía de acuerdo a la estabilidad de ingresos de los padres; por ejemplo, en varios casos, 
los padres de los entrevistados eran profesores y su actividad no se detuvo durante la pandemia. En otros casos, la principal fuente de ingresos han sido los negocios familiares, como, por ejemplo, el restaurante de la madre de una entrevistada, que no detuvo sus actividades gracias al delivery o el caso de un hotel que continuó funcionando durante la pandemia.

La interacción entre las dinámicas del hogar y las dinámicas estudiantiles de los jóvenes dependen de los factores ya mencionados, pues algunos resultaron más afectados que otros. Para ahondar en estas afirmaciones pasaremos a trabajar los casos encontrados. En primer lugar, en el caso de Anthony -originario de Cañete- él considera que sus estudios no se vieron afectados por la pandemia. El hecho de que sus padres hayan continuado trabajando como profesores durante la crisis sanitaria, le permitió costearse sus estudios e, incluso, mantener su estadía en Lima, aún a pesar de que dejó de trabajar en sus prácticas pre profesionales. Antes de la pandemia, su motivación para trabajar radicaba, más bien, en el deseo de reducir los gastos de sus padres destinados a su estadía en Lima. Asimismo, su viaje de retorno a Cañete estuvo motivado por su deseo de disminuir los gastos de sus padres al ser innecesaria su estadía en Lima por la educación virtual. Ya una vez en Cañete, Anthony empieza a considerar que puede estudiar más tranquilo, pues realiza sus actividades durante la noche sin que sus padres lo interrumpan. Por otro lado, los estudios de Katherine -originaria de Vijus, Pataz-, sí se vieron afectados por la pandemia, pero por problemas de conexión de internet principalmente, lo cual será abordado posteriormente. Ahora bien, su madre es dueña de un hotel en Pataz - La Libertad y Katherine ha trabajado ahí desde que regresó a su hogar en abril del 2020. El hotel siguió funcionando durante la crisis, debido a que muchos trabajadores de minas aledañas llegaban a hospedarse. Katherine indica que recibe el salario mínimo mensual y que este trabajo le va a servir para su futuro como administradora. Asimismo, el trabajo en el hotel no interfiere con sus deberes académicos, ya que tiene un horario flexible y ella se considera una persona responsable. Por último, tenemos el caso de Rosa, cuyos estudios se vieron interrumpidos por retraso de la universidad. Durante el tiempo libre realizó delivery para el restaurante de su madre. Al preguntarle si siguió haciéndolo durante las clases, respondió que ella "lo hacía cuando podía" ya que su principal labor era estudiar y su madre también estaba consciente de eso.

Ahora bien, la situación de nuestros entrevistados es diversa, por lo cual el poco grado de conflicto entre los estudios y las responsabilidades familiares de los casos antes mencionados, no se cumple para todos los jóvenes. Los entrevistados mencionados anteriormente no tienen la obligación de trabajar, lo cual está condicionado por la posición económica de su familia. Hay otro grupo de jóvenes que, al regresar a sus lugares de origen han encontrado distintas responsabilidades domésticas y laborales que los desconcentra y les quita tiempo para estudiar pues "en el campo siempre hay algo que hacer". Entre las nuevas responsabilidades de los estudiantes están el cocinar, darle de comer a sus hermanos, alimentar a los animales varias veces al día, atender la tienda familiar y trabajar las tierras familiares, en donde la actividad, 
frecuencia y dificultad varía en cada caso. En muchas situaciones, los estudiantes deben interrumpir sus clases cuando sus padres les piden ayuda: cuando hay algún cliente que atender, cuando sus padres tienen que salir y dejar a alguien a cargo, o cuando simplemente hay mucho trabajo y se necesitan más manos en la chacra. Un sentimiento general que sienten los estudiantes es el de agotamiento por la suma del trabajo en el campo y las responsabilidades educativas, pues, según Karen (Cusco, 22 años), es "algo que no existía en la ciudad, donde solo tenía tareas como ordenar mi cuarto o limpiar".

Al conversar con las entrevistadas, se evidenciaba que su retorno había generado que retomen ciertas labores de cuidado frente a sus padres y otros familiares. Por ejemplo, Marcy -originaria de Chiquián- retomó la responsabilidad de la cocina y del cuidado de las mascotas al volver a su casa, al igual que distintas labores de cuidado hacia sus seres queridos. De igual manera, Deysi -originaria de Caraztomó la responsabilidad de la cocina diaria, responsabilidad que se volvió una obligación principal incluso al reiniciar sus clases a distancia: "Bueno, ahora hago los quehaceres de la casa, tengo que hacer el almuerzo y eso es todos los días [incluyendo los días de clases]" (Deysi, 20 años).

Como se percibe en la anterior cita, la responsabilidad de Deysi en la cocina se acrecentó al momento de reanudar las clases debido a un acuerdo con su familia, un acuerdo con el cual ella, en realidad, no está muy conforme. Por último, en el caso de Grecia -originaria de Chiquián-, la cocina y la limpieza no han formado parte de sus responsabilidades constantes, pero sí la compra de alimentos para cocinar y el cuidado de sus abuelos. Estas responsabilidades han chocado con sus clases virtuales y comenta que se le hace difícil poder ayudar constantemente. Sin embargo, ella muestra una fuerte conciencia acerca de su responsabilidad con sus abuelos. Retornó a Chiquián específicamente para hacerles compañía y cuidar de ellos. Cuando habla sobre el futuro menciona que si tiene la posibilidad de mantener las clases virtuales lo hará en favor de mantenerse en Chiquián con sus abuelos, a pesar de los problemas que ha tenido al juntar sus responsabilidades familiares y académicas. Otra situación similar es la de Tabita - de Huánuco-, quien tiene, también, a su responsabilidad la labor de cuidado de su menor hijo. La dificultad de conciliar sus labores de cuidado y labores domésticas con sus estudios universitarios la llevaron a realizar una estrategia de doble residencia durante la pandemia. De este modo, cuando tiene clases virtuales viaja a la ciudad de Huánuco donde convive con su pareja. Allí se dedica ella sola al trabajo de cuidado de su hijo y de su casa, mientras su esposo se dedica al trabajo como electricista. En temporada de exámenes o cuando debe hacer trabajos finales, ella viaja hacia Chavín de Pariarca, zona rural donde reside su madre y donde recibe su apoyo con el trabajo de cuidado mientras ella se dedica a sus exámenes. No obstante, allá también colabora con las labores domésticas y agrícolas cuando tiene tiempo disponible.

En cuanto a los varones, también se evidencia la toma de responsabilidades al retornar a los hogares, pero de diferente carácter. Las responsabilidades masculinas 
se suelen realizar fuera del hogar. En el caso de Cristian -originario de Cascapara-, estas se realizaban en la chacra de palta y en el taller de estructuras metálicas de su padre. En su caso, las labores de cuidado se muestran con sus hermanos menores, como en el siguiente fragmento de la entrevista:

Antes de las clases virtuales mis días eran ayudar a mi papá en su taller y en regar las plantas, repasaba algunos temas de las clases anteriores, jugamos con mis hermanos y también en algunas horas revisaba las redes sociales (Cristian, 25 años).

En el caso de Héctor -originario de Barranca-, su responsabilidad principal era ayudar a su padre en la atención de una de sus tiendas en Barranca. Ambos participantes, Cristian y Héctor, han mencionado que también apoyaban en las labores domésticas dentro de la casa, como en la limpieza y la cocina, pero estas no eran responsabilidades totalmente suyas, sino compartidas en familia.

Al respecto de las labores de cuidado, no solo se retoman en una dirección -de jóvenes hacia familiares que residen en el lugar de origen-. También se ha notado que el cuidado se restablece desde la familia hacia los jóvenes. No obstante, al depender nuevamente del cuidado de sus padres, se establece también una autoridad sobre los jóvenes. En el caso de Grecia, esta autoridad se expresa a través de las llamadas de atención que recibe cuando se amanece por los trabajos de la universidad o cuando se demora o no llega a las comidas en familia. La autoridad también se manifiesta en la responsabilidad asignada por la madre de Deysi, ya que debe encargarse del trabajo en la cocina pese a que ella no esté de acuerdo, pues dificulta sus actividades universitarias y no es de su agrado.

Ahora bien, a pesar de las dificultades mencionadas anteriormente, un grupo de los jóvenes entrevistados logran rescatar una sensación positiva por la nueva interacción con la familia y amigos, con quienes no habían pasado mucho tiempo en los últimos años. Los estudiantes manifiestan que este cambio les ha permitido acercarse a su familia en múltiples dimensiones, como, por ejemplo, han podido conocer a sus hermanos a mayor profundidad, apoyar a sus padres, compartir comidas con la familia, "en comparación con la soledad en la ciudad" (Karen, 22 años), lo cual genera una sensación de satisfacción y gratitud en ellos.

Además, los participantes mencionan el apoyo que reciben de parte de sus familias en esta nueva convivencia. En primer lugar, el encuentro cotidiano entre las dinámicas familiares y las dinámicas estudiantiles genera un proceso de conocimiento y comprensión de parte de la familia. Este aprendizaje ha sido positivo desde laperspectiva de Deysi, puesto que su familia ha llegado a entender mejor sus responsabilidades y a respetar su organización, aunque este proceso haya sido complejo. Por otro lado, Marcy percibe que las relaciones familiares y la vida universitaria han encajado de manera más positiva en su caso, puesto que sus padres le dan espacio para estudiar y la alimentan mejor. Esta percepción la comparten, en parte, Grecia y Cristian. Los 
tres sienten que una ventaja de la nueva convivencia ha sido el cuidado que reciben de sus padres; principalmente, el apoyo moral y la preocupación por su alimentación, tema que era un problema para Grecia y Marcy cuando vivían solas. En este sentido, sienten que este cuidado ha sido un apoyo a su vida académica también. De manera semejante, Tabita señala como positivo el apoyo de su madre en las labores de cuidado de su hijo mientras ella estudia. Asimismo, valora positivamente el hecho de que no debe dejar a su hijo al cuidado de una persona ajena a su familia mientras ella estudia en la universidad. Señala que lo más probable es que hubiera tenido que dejar a su hijo en una guardería para poder continuar sus estudios.

En cuanto a la relación entre pares, Rosa explica que siempre ha estado en contacto con sus amigas y que estar reunidas nuevamente ha significado mucho para ella. Además, en Chacas-Huaraz, las restricciones sociales no son muy severas, por lo cual puede verlas: "Acá el aire es más limpio. Ahora puedo salir a caminar, he salido con algunas amigas [...] la mayoría de personas tiene casas grandes con patios, zonas libres. Se experimenta diferente el estar encerrado" (Rosa, 22 años). De igual modo, Kevin -de Huánuco- señala como aspectos positivos el poder respirar aire fresco y no tener que estar encerrado. A ello, le añade la vida ajetreada en Lima y la gran cantidad de gastos que uno debía realizar tanto para comer como para transportarse. En Chavín, en cambio, los alimentos son obtenidos de la propia producción agrícola familiar y local, mientras la movilidad puede realizarse a pie o en moto, sin inconvenientes.

Como se puede observar, la pandemia ha forzado una nueva interacción familiar en estos casos, que contrasta con la experiencia de libertad de los estudiantes en la ciudad con la nueva convivencia familiar. Son muy pocos los entrevistados que han podido mantener como única prioridad el estudio, muchos de ellos han incorporado a sus rutinas obligaciones de sus familias como el trabajo agrícola, el trabajo en negocios familiares y el trabajo de cuidado. Por otro lado, la perspectiva negativa de los cambios, para los entrevistados, implica, principalmente, los choques entre las rutinas y cómo estos han podido causar desencuentros con la familia que han desatado discusiones o resentimientos entre los distintos miembros. Notamos, también, que estos conflictos se resuelven, en mayor medida, a favor de los familiares cuando existe una jerarquía familiar marcada. En esos casos, los jóvenes no pueden evadir o "negociar" las nuevas responsabilidades que se les han presentado, debido a que se encuentran en la casa de sus familias (abuelos, padres) y, al estar al cuidado de estos, los jóvenes se ven obligados a retribuir. Sin embargo, a pesar de estas tensiones, este cuidado ha resultado positivo para los jóvenes estudiantes: regresar a su hogar les ha permitido acercarse nuevamente a su familia, interactuar con ellos en mayor profundidad y ser apoyados por sus padres.

\section{Virtualización de la educación: estrategias y desafíos}

La alerta sanitaria por coronavirus ha reforzado las persistentes desigualdades educativas en el Perú. Debido a la virtualización masiva de los servicios educativos, 
el acceso a estos ha sido restringido a un grupo mayoritario de estudiantes. Según el INEI (2020), el acceso a Internet, durante el primer trimestre del 2020, alcanzó al $40,5 \%$ de los hogares del resto urbano y al 5,9\% de los hogares del área rural. En este marco, las desigualdades sociales preexistentes han agudizado distintas dificultades, como la mencionada, en el contexto de la crisis sanitaria con las que los estudiantes han tenido que lidiar- específicamente, frente a la virtualización de la educación.

Antes de la pandemia, los estudiantes accedían a internet mediante la señal gratuita del campus universitario o mediante planes de datos en su teléfono o alquilando internet de sus arrendatarios. Sin embargo, en el campo, los entrevistados reportan que hay un problema generalizado de falla en la señal de teléfono e internet que les impide llevar sus clases virtuales adecuadamente. Muchos de ellos no contaban con internet fijo en sus casas antes de la pandemia, sino solamente el internet de sus celulares, pero este no era suficiente para llevar una clase sincrónica sin interrupciones, revisar el correo o subir archivos a diferentes plataformas en línea. Los estudiantes cuentan que hay partes de sus distritos donde simplemente no hay señal de ningún tipo y, si es que llega la señal, esta está limitada a ciertas zonas y puede irse por momentos de manera repentina, incluso teniendo un plan de internet. Factores impredecibles como las lluvias, los vientos fuertes y las tormentas pueden hacer que la señal se vaya por varias horas y, en el peor de los casos, que la energía eléctrica se corte por días, impidiendo que puedan cargar la batería de sus dispositivos, además de quedarse sin señal.

Estas dificultades de conexión son difíciles de superar, incluso cuando los jóvenes tienen el apoyo económico de sus padres. Por ejemplo, Katherine abandonó el ciclo 2020-1, debido a que no llegaba un buen internet hasta su casa; como ella indica: "Ya sabía que el internet fuera de Trujillo era malo por eso decidí no estudiar". En agosto, decidió volver a matricularse para no perder todo el año y su mamá empezó a comprarle "megas" de internet para que sus clases no se vean interrumpidas por problemas de conexión. Sin embargo, para ella es muy difícil aprender de esta manera y expresó que, a veces, se arrepentía de haber estudiado el ciclo 2020-2, ya que la señal seguía siendo muy mala.

Gabriel -de Huaraz-, estudiante de Arquitectura de 19 años, conoce a mucha gente que tuvo que dejar de estudiar el primer ciclo de pandemia. A pesar de que él no tiene dificultades de conexión, manifiesta que "algunos cursos son difíciles, ya que tienes que mandar fotos de algunos dibujos y la resolución de algunas cámaras no es la mejor". Por tanto, existen ciertos cursos o carreras cuya virtualización resulta más complicada, sobre todo aquellos prácticos que requieren de trabajo aplicado. Por ejemplo, Rosa, estudiante de arquitectura se muestra preocupada, ya que está perdiendo clases prácticas debido a la prolongación de la virtualidad. Explica que, si en el presente año, 2021, las clases se siguieran dando de manera remota, perdería un año de clases, lo cual significaría un gran atraso. Tal situación es similar a la experimentada por Kevin -originario de Huánuco--, estudiante de 
mecánica automotriz, quien tuvo que dejar sus estudios los dos ciclos del 2020, tanto por problemas económicos que le impedían continuar con el pago de sus clases, como por la imposibilidad de llevar de manera virtual la mayoría de cursos que le faltaban para culminar su carrera, al ser esta una carrera técnica y de aprendizaje aplicado. Situación distinta fue la experimentada por Anthony, quien cuenta con una computadora propia, y se encuentra en los últimos ciclos de la carrera de ingeniería de sistemas, por lo cual no requería conectarse constantemente a clases. Además, esta especialidad tiene la ventaja de que sus cursos son plenamente virtualizables.

Para los problemas de conexión recurrentes, algunos jóvenes han encontrado mecanismos para tratar de continuar con sus estudios. Para tal fin, es importante contar con distintas redes de apoyo, ya sean comunitarias o familiares. En el caso de la comunidad campesina de Coñamuro en Urcos, Cusco, donde vive Alma, se logró instalar una antena de conectividad tras mucha insistencia de los dirigentes comunales hacia la municipalidad. En el distrito de Chacas, Áncash, donde vive Rosa, pasó algo parecido: un grupo de estudiantes, incluyéndose ella, y otros pobladores que necesitaban la conexión a internet con fines laborales, se reunieron con el alcalde para pedir que se instale una nueva antena y se mejore la conectividad, lo cual se logró a los pocos meses de hacer el pedido. El caso de Tabita, en cambio, muestra cómo las redes familiares a distancia pueden servir también para lidiar con este problema, pues cuando se dirige a Chavín buscando recibir apoyo de su madre con el cuidado de su hijo, llama a su primo quien está en la ciudad para que le ayude con la subida de archivos debido a la mala conexión de internet en Chavín.

En cuanto a la relación con los profesores, algunos de los entrevistados indican que esta se ha vuelto problemática, en algunos casos, por falta de comunicación con los estudiantes, porque piensan que los alumnos se salen de las clases sincrónicas por desinterés, cuando en realidad es por problemas de conexión. Por ejemplo, Rosa comenta que al empezar sus clases en julio del 2020, "los profesores no entendían los problemas con el internet de los alumnos". De igual manera, Tabita señala haber desaprobado un curso por problemas de conexión. Pese a que se lo explicó al profesor, este resultó totalmente intransigente. Sin embargo, en otros casos, los profesores se muestran comprensivos, como comenta Gabriel, pues a pesar de la dificultad que representa el que su carrera sea práctica, los profesores han dado facilidades para que esta se desarrolle lo más amigable posible.

Por otro lado, las metodologías de evaluación virtual son percibidas por Alma (Cusco, 24 años), Karen (Cusco, 22 años), Kelvin (Amazonas, 18 años) e Isaac (Cusco, 26 años) como injustas, ya que la poca conexión impide que, en ocasiones, los trabajos se envíen adecuadamente y que sus respuestas se guarden por el colapso de las plataformas. Además, los límites de tiempo impiden entregar respuestas después de la hora o que algunos estudiantes puedan compartir respuestas y hacer los exámenes en grupo, lo cual perjudica a los que sí estudiaron. Las plataformas empleadas han recibido bastantes críticas porque se saturan, se cuelgan y perjudican el desarrollo de la clase, de modo que a veces las clases se tienen que suspender y posponer. 
Otro factor que dificulta el desarrollo de las clases virtuales es la distracción. Algunos de los jóvenes entrevistados no encuentran un espacio de tiempo que puedan dedicar totalmente a los estudios, pues la familia tiende a hacer ruido, poner música, llamarlos para hacer alguna tarea, cocinar. En general, la interacción doméstica en medio de un ciclo de estudios, que antes de la pandemia era reducida o nula, les resulta una gran distracción, en comparación a la concentración que podían obtener en las habitaciones que alquilaban, de manera individual, en las ciudades. Deysi, por ejemplo, indica que estar con su familia en casa le ocasiona distracciones al momento en que se conecta a sus clases, debido a que hacen ruido. Sumado a esto, cuando ella les pide que bajen el volumen, estos se incomodan ya que no pueden conversar o ver la televisión como lo hacían antes.

Además, como se mencionó en la sección anterior, las nuevas responsabilidades para los jóvenes, surgidas a raíz de la pandemia, generan una distracción, también, respecto al desarrollo de las clases, como lo expresa en el siguiente fragmento de la entrevista de Grecia:

[...] Los altercados que sí ocurrieron es cuando tengo muchas cosas que hacer y mi abuelita o mi abuelito a veces quieren que les apoye con algo, porque como ya están viejitos, a veces mi mamá se demora en comprar en la calle o en otra cosa, ellos me piden ayuda y yo a veces quiero ayudarlos pero estoy haciendo, estoy prestando atención en la clase o estoy haciendo alguna práctica, algún examen, y ellos como que se resienten o se molestan a veces pero yo les digo que es por eso, que no es que no quiera hacerlo. [...] (Grecia, 18 años).

Además, Grecia comenta que, a veces, se generan problemas por el uso de espacios y herramientas compartidas. Otro aspecto mencionado por ella fueron los problemas de internet, que alguna vez han provocado incomodidad con su familia. Finalmente, las clases virtuales generan dificultades en la misma interacción familiar, pues señalan que no pueden pasar tanto tiempo con ellos al dedicarse a sus clases la mayor parte del tiempo.

Es así que este panorama ha demostrado que tener una conexión a internet es un privilegio de unos pocos, en lugar de un derecho básico, necesario, para acceder a la educación. Durante la crisis sanitaria, todos los estudiantes entrevistados han tenido dificultades con la educación virtual que los ha puesto en desventaja con respecto a sus pares de áreas urbanas. Estas dificultades han ido desde problemas para el correcto desarrollo de las clases hasta la deserción estudiantil. Asimismo, la virtualidad ha implicado un reto para los mecanismos de evaluación, ya que para algunos aumenta la facilidad de plagio y con ello, la percepción de injusticia. Igualmente, estas dificultades se alivian o se agravan en función del grado de comprensión que el profesor tenga con los alumnos. Además, las distintas responsabilidades e interacciones familiares conllevan a los jóvenes a distraerse durante el horario de clases, sumado a las disputas en torno al espacio y tiempo doméstico. No obstante, 
frente a estas dificultades, sobre todo la de conexión a internet, los jóvenes han recurrido a diversas estrategias que les permitieron solucionar estos problemas, sobre todo a través del uso de redes familiares o comunales.

\section{Proyectos de vida: cambios y permanencias}

Casi todos los entrevistados, a excepción de uno, han mantenido sus proyectos de vida y una actitud de optimismo frente al futuro. Esto puede ir en contra de lo esperado según el informe de diagnóstico "Los jóvenes y la COVID-19" (2020), en el que se señala que primaría un ambiente de incertidumbre, debido a la situación de vulnerabilidad social de la mayoría de jóvenes (OIT, 2020). No obstante, en el mismo informe se señala que esto sería vivido de manera más intensa por aquella población juvenil que haya visto interrumpidos sus estudios (OIT, 2020). Además, los grados de vulnerabilidad (exposición a problemas de salud, desempleo, endeudamiento) dependen de la mayor o menor cantidad de activos con los que cuentan, entre los cuales se consideran no sólo la propiedad y la educación, sino también el capital social (CEPAL, 2016). Este último factor parece ser el que ha permitido una mayor adaptabilidad a la condición de la crisis, que permite a su vez mantener los proyectos de vida.

En ese sentido, para los entrevistados, sobresale como un proyecto importante la continuación de los estudios, el logro de estabilidad laboral y la posibilidad de emprender negocios propios que le permitan un mayor grado de autonomía, como elemento central en sus proyectos de vida. Además, estas aspiraciones están vinculadas a la vida en espacios urbanos y, en ciertos casos, a la migración internacional como modo de seguir "progresando". Por ejemplo, Rosa (22 años) espera ir a estudiar la maestría al exterior: "Antes no me interesaba esto, fue poco a poco. Estando acá en Chacas empecé a buscar becas”. Por su parte, Gabriel (19 años) tiene un plan parecido: "Cuando termine la carrera, encontraré un trabajo, quiero viajar. Me gustaría conocer Italia”.

Ahora bien, algunos entrevistados no descartan regresar a sus lugares de origen cuando sean profesionales. El plan de Katherine (24 años) es terminar sus estudios lo más pronto posible y regresar a Pataz para administrar el hotel de su madre, donde trabaja actualmente: "Quiero implementar nuevas cosas. Si me ofrecen trabajo fuera de La Libertad, lo pensaría, [...] probablemente terminaría tomándolo." A Rosa le gustaría regresar a Chacas en un futuro lejano: "Sí me gustaría regresar y contribuir en el pueblo [...] proyectos de urbanismo, vivienda.". Por su parte, a Kevin y Tabita les gustaría mantener su vivienda en Chavín cuando ya sean profesionales para mantener sus chacras como una fuente de ingreso adicional -encargándosela a algún peón- y como lugar de reposo. Las tres situaciones, no obstante, muestran diferencias. En el primer caso, el deseo de retorno de Katherine se debe a que existen oportunidades para crecer económicamente y profesionalmente -ganar experienciaposibilitadas a su vez por la minería en la zona. En el segundo caso, la posibilidad 
es más remota pero existe, aunque no depende de un capital propio como en el caso de Katherine, sino de la inversión estatal o municipal. Asimismo, su retorno estaría en conexión con su carrera, por lo que sería parte de su desarrollo laboral. Finalmente, en el tercer caso, dado que no existe vinculación entre las carreras de los entrevistados -mecánica automotriz y contabilidad-y las oportunidades de empleo en la zona -principalmente agrícola-, estos ven en sus lugares de origen solo una fuente de ingresos secundaria y un lugar de descanso.

En suma, casi todos los entrevistados mantuvieron sus proyectos de vida a largo plazo y su optimismo respecto a que la situación mejorará en el futuro para retomar sus actividades previas a la crisis. Un caso excepcional sería el de Kevin, quien -como se señaló anteriormente- suspendió sus estudios técnico-superiores a raíz de la pandemia, tanto por problemas económicos, como por la dificultad de la virtualización. Precisamente, él muestra una mayor incertidumbre con respecto a qué hacer en el futuro, pues señala no saber si vale la pena retomar sus estudios o, en lugar de ello, trabajar para acumular un capital económico suficiente que le permita poner su propio negocio. No obstante, mantiene el optimismo respecto a que podrá migrar para continuar con cualquiera de los dos caminos que decida.

Un punto a recalcar al respecto es el mantenimiento del "ideal emprendedor" en los planes a futuro de algunos de los entrevistados, puesto que señalaron como principal proyecto de vida la autonomía económica (un negocio propio), y la pandemia no ha representado un cambio radical en la búsqueda de estos objetivos. En el caso de Marcy, ella planea, en un aproximado de 10 años, establecer su propio negocio en Huaraz o Lima. Christian y Deysi presentan planes similares, aunque aún no sepan qué tipo de negocio establecerán, la búsqueda de autonomía económica sigue en pie. Tabita planea terminar sus estudios en contabilidad para tener habilidades que le permitan desarrollar su propio negocio. Anthony, próximo a egresar en ingeniería de sistemas, también muestra un plan similar: trabajar como asalariado y acumular el capital suficiente para establecer su propia empresa relacionada a su carrera. De esta manera, la carrera que estudian es vista como un medio o recurso para conseguir capital y experiencia que les permita realizar dicho objetivo. Por su parte, a pesar de que Kevin suspendió sus estudios y sus proyectos a corto plazo se han reformulado, su ideal de autonomía económica no ha cambiado, puesto que la carrera era solo un medio para ese fin. Además, su indecisión respecto a si retomar o no sus estudios se relaciona con las posibilidades que ofrece la minería informal en Chavín (Huánuco) para conseguir dinero rápidamente, aunque de manera muy riesgosa.

Si bien es cierto, como hemos mencionado, hay continuidades en los planes del proyecto de vida a largo plazo de cada estudiante, los entrevistados consideran este tiempo de retorno como una "pausa" a dichos planes. Pero, al mismo tiempo, ven esta etapa como un momento de aprendizaje. En ese sentido, los jóvenes también han utilizado este tiempo para repensar sus valores y cambiar su autopercepción. De esta manera, consideran que la pandemia les ha permitido reconsiderar la importancia de 
la familia en su vida, así como del ahorro. Los mismos jóvenes se perciben, ahora, como más responsables y maduros, debido a la crisis experimentada y a la serie de dificultades a las que le hicieron frente. Sus planes inmediatos han sido repensados y han cobrado un mayor sentido de responsabilidad hacia el futuro, lo cual ha hecho que se planteen, de manera más contundente, metas a mediano y largo plazo: "Hace un año tenía los objetivos menos claros. La situación es distinta ahora, el tiempo pasa y no por gusto [...] Ahora sé lo que quiero y lo que tengo que conseguir, antes estaba como viendo que pasaba" (Rosa, 22 años). Para Katherine, por su parte, este tiempo le ha traído importantes lecciones de vida: "Ahora puedo manejar mis emociones, actitudes. He aprendido a vivir sin miedo y afrontar retos. No perder oportunidades, aprovecharlas y manejar bien el dinero". En ese sentido, la crisis económica ha suscitado que los jóvenes sean conscientes de la importancia de los ingresos fijos y las consecuencias de las deudas, como indica Rosa: "Mis papás no tenían ahorros, ellos trabajan y lo invierten para algo. Ahora me doy cuenta de lo importante que es tener un ingreso extra o un ahorro. Te saca de apuros."

\section{Conclusiones}

Este trabajo exploratorio buscó conocer cómo se verían afectados los proyectos de vida de jóvenes universitarios a partir del impacto de la pandemia. En un principio, se consideró que la pandemia y la situación de migración de retorno a espacios rurales implicaría un estancamiento y un quiebre en los planes a futuro de los jóvenes estudiantes de educación superior, así como un sentimiento de incertidumbre y frustración generalizados. Sin embargo, tal impacto, a pesar de implicar ciertos cambios, no generó una transformación profunda en sus proyectos de vida ni la existencia de una marcada incertidumbre, puesto que los jóvenes recurrieron a distintas estrategias que les permitieron adaptarse a la nueva situación adversa, en función de los capitales-sociales, económicos, culturales-con los que estos contaban. Por lo tanto, la crisis sanitaria vivida, y las consecuencias de esta, no generaron un estancamiento ni un cambio fundamental en los planes de los jóvenes participantes. Lo que encontramos fue una "pausa" a su vida autónoma en las distintas ciudades donde estudiaban, mas no a sus planes a futuro.

En ese sentido, si se tiene en consideración el nivel de autonomía desarrollado en los años en que los jóvenes vivieron en las ciudades, podemos notar que el regreso al hogar familiar puede significar una "pausa" a los distintos aprendizajes y al ritmo adquirido durante este periodo. En ciertos aspectos esto es realmente lo que ha sucedido. El retorno al hogar familiar ha significado el regreso a la dependencia de los padres y familiares, sobre todo en los aspectos de las labores de cuidado de padres a hijos e hijas. También notamos esto en el nivel de autoridad que los padres retoman sobre sus hijos e hijas, sobre todo teniendo en cuenta que el retorno no fue totalmente voluntario, sino forzado por la situación de pandemia. Sin embargo, no se podría considerar que la transición de los jóvenes a su adultez se haya "pausado"; por el contrario, esta ha seguido su curso por medio de otras vías. Los entrevistados 
asumen nuevos compromisos a la par de la familia, sobre todo en el trabajo familiar y las labores de cuidado. Asimismo, la crisis a raíz de la pandemia ha generado un sentimiento de responsabilidad respecto a sus propias vidas, pues se consideran más "maduros" y con una visión más centrada tanto en "hacerse cargo de su propio futuro" como en la importancia de la familia.

En ese sentido, las redes familiares fueron un factor clave como estrategia para enfrentar las adversidades. Por un lado, el viaje de retorno no significó un proceso tan riesgoso para los entrevistados, debido, sobre todo, al apoyo de sus familiares, tanto en lo monetario como en el transporte. Por otro lado, contaban con un lugar establecido al cual iban a llegar, donde encontrarían hospedaje y alimentación. No obstante, a pesar de la importancia de las redes familiares, estas también implican obligaciones para los jóvenes - diferenciadas según el género- que pueden generar conflicto con sus actividades educativas y autonomía personal. Es así que las estudiantes mujeres han asumido, en su mayoría, labores domésticas y de cuidado; mientras que los estudiantes varones se dedican, sobretodo, a labores agrícolas o negocios u oficios de los padres. Sin embargo, estas responsabilidades fueron exigidas, en mayor o menor grado, dependiendo del tipo de actividad económica de los padres. Así, entre aquellos padres dedicados a la agricultura y ganadería, la demanda por ayuda era mayor, al ser una actividad que exige mayor mano de obra. En casos donde los padres tienen otros oficios, como el de profesores o conductores, esta exigencia era menor.

Además de ello, la ocupación de los padres influyó en el grado de impacto que la pandemia tuvo sobre los entrevistados. Por un lado, las familias cuya principal actividad es la agrícola, pudieron seguir subsistiendo gracias a la producción para el autoconsumo -o al intercambio local- y al menor costo monetario de la vida en el campo. Por otro lado, los padres que tenían un negocio propio en el sector de servicios (hoteles, restaurantes) siguieron recibiendo ingresos monetarios durante la crisis sanitaria, pues siguieron funcionando a pesar del confinamiento por la COVID-19. Asimismo, los padres que eran asalariados y trabajaban como profesores mantuvieron sus actividades. Esto permitió que casi todos continúen con sus estudios de manera virtual.

Ahora bien, la virtualización también implicó retos que trascendieron el apoyo económico familiar, como fueron los problemas de conectividad. Estos se debían a problemas con la señal de internet, la cual era limitada en algunas zonas o, simplemente, se perdía en mitad de una clase. No obstante, en algunos pocos casos, pudieron recurrir a ciertas medidas para contrarrestar esos problemas. En estos casos, se recurrió también al apoyo de redes sociales, ya sea para solicitar a la municipalidad con la presión comunal que solucione estos problemas de conectividad o para solicitar ayuda a parientes lejanos con mejor conexión para realizar el envío de tareas virtuales. Asimismo, durante su jornada, los jóvenes tuvieron que lidiar con las distintas responsabilidades del hogar antes explicadas que les generaron 
distracciones e interrupciones. A ello se le suma los conflictos por el uso del espacio así como por los dispositivos compartidos con otros miembros de la familia. Los ruidos generados por los familiares eran también un factor de distracción.

En cuanto a la dimensión más personal de los estudiantes, se puede encontrar una valoración positiva como resultado de la nueva convivencia con sus familiares, en tanto les ha permitido entablar una relación más cercana con sus padres y hermanos que no hubiera sido posible en circunstancias diferentes, así como poder apoyar en las labores productivas familiares. A pesar de que las nuevas responsabilidades domésticas y laborales asumidas construyen en algunos casos distracciones respecto a las clases virtuales, prevalece una sensación de satisfacción por compartir y apoyar a su familia. Los jóvenes entrevistados piensan en el regreso a sus lugares de origen como una medida temporal por la pandemia. En ese sentido, esperan regresar a sus lugares de estudio y trabajo cuando la crisis haya pasado y las restricciones sociales hayan disminuido. Al respecto,los jóvenes ven este tiempo como de aprendizaje y mantienen una visión optimista del futuro, lo cual ha conllevado a una permanencia en sus proyectos de vida. La mayoría, apoyados en la seguridad de sus estudios o en capital económico que esperan conseguir en un futuro próximo.

Finalmente, es importante reconocer que la mayoría de entrevistados en este artículo se distinguen mucho de otros jóvenes, menos privilegiados, cuyas familias tienen menos capital económico y cultural. Por ejemplo, en el estudio realizado por Burneo y Trelles (2020) en el Alto Piura, se afirma que los retornantes han tenido que replantear sus planes y proyectos de vida, tanto a corto como a mediano plazo. El regreso de estos jóvenes ha implicado un mayor nivel de incertidumbre, incluyendo principalmente el abandono momentáneo de sus estudios y el retorno a actividades agrícolas. Entre los casos trabajados en este artículo, el que más se asemeja a la situación planteada por Burneo y Trelles es el de Kevin, puesto que también sufrió un mayor nivel impacto y muestra mayor incertidumbre a raíz de la pandemia. Esto debido a que sus padres cuentan con menor capital económico y cultural (su padre posee un taller mecánico que recibe bajos ingresos y su madre se dedica a actividades agrícolas), lo que lo forzó a interrumpir sus estudios y replantear sus proyectos. 


\section{Referencias bibliográficas}

Azaola, C. (2012). "Becoming a migrant: aspirations of youths during their transition to adulthood in rural Mexico". Journal of Youth Studies, 15(7), 875-889, DOI:10.1080/136762 61.2012 .677813

Bourdieu, P. (2000). Poder, Derecho y Clases Sociales. Desclée.

Burneo, M. y Trelles, A. (2020). Migración de retorno en el Alto Piura en el contexto de la pandemia por la Covid-19. CIPCA.

http://www.cipca.pe/sites/default/files/documents/files/Investigaci\%C3\%B3n $\% 20$ Retornantes.pdf

CEPAL, N. (2016). Desarrollo social inclusivo: una nueva generación de politicas para superar la pobreza y reducir la desigualdad en América Latina y el Caribe.

Chambers, R. y Conway, G. (1991). Sustainable Rural Livelihoods. Practical Concepts for the 21st Century. Brighton: IDS.

D’Angelo, O. (2004). Proyecto de vida como categoría básica de interpretación de la identidad individual y social. Revista cubana de Psicología, 17(3), 270-276. http://bibliotecavirtual. clacso.org.ar/Cuba/cips/20120827125359/angelo8.pdf.

Harvey, D. (2007). Breve historia del neoliberalismo. Akal.

Instituto Nacional de Estadística e Informática (INEI). (2019). Indicadores de educacion por departamento 2008-2018. https://www.inei.gob.pe/media/MenuRecursivo/publicaciones digitales/Est/Lib1680/libro.pdf

Instituto Nacional de Estadística e Informática (INEI). (25 de junio del 2020). El 40,1\% de los hogares del país tuvo acceso a internet en el primer trimestre del 2020. http://m.inei. gob.pe/prensa/noticias/el-401-de-los-hogares-del-pais-tuvo-acceso-a-internet-en-el-primertrimestre-del-2020-12272/

Koc, N. (2001). "Minería, economía y racionalidad: transformaciones en la comunidad campesina de Huachocolpa, Huancavelica-Perú". Lima: PUCP. Tesis de Licenciatura en Antropología.

Lamas, L. (2017). "Somos una raza distinta que puede lograrlo todo": emprendimiento, educación y nuevas concepciones raciales en el Perú neoliberal. Racismo y lenguaje. Lima: Fondo Editorial de la Pontificia Universidad Católica del Perú.

https://opencommons.uconn.edu/cgi/viewcontent.cgi? referer=https://www.google. com/\&amp;httpsredir=1\&amp;article=1001\&amp;context=facpubworks

Organización Internacional del Trabajo (OIT). (2020). Los jóvenes y la COVID-19: Efectos en los empleos, la educación, los derechos y el bienestar mental. https:/www.ilo.org/wcmsp5/ groups/public/---ed_emp/documents/publication/wcms_753054.pdf 
Proyecto CRECER. (2020). La escuela rural: el reto de adaptar estrategias para un país diverso $y$ desigual. https://saludconlupa.com/noticias/la-escuela-rural-el-reto-de-adaptarestrategias-para-un-pas-diverso-y-desigual/

Punch, S. (2015). "Youth transition and migration: negotiated and constrained interdependencies within and across generations". Journal of Youth Studies, 18 (2), 262-276. http://dx.doi.org/10.1080/13676261.2014.944118

Urrutia, A. y Trivelli, C. (2018). Geografias de la resiliencia: la configuración de las aspiraciones de los jóvenes peruanos rurales. https://repositorio.iep.org.pe/bitstream/ IEP/1130/3/Trivelli-Carolina_Urrutia-Adriana_Geografias-resilencia-configuracionaspiraciones-jovenes-peruanos-rurales.pdf 